\title{
Dynamic polarizability of Rydberg atoms: Applicability of near-free electron approximation, gauge invariance and the Dirac sea
}

\author{
Turker Topcu and Andrei Derevianko \\ Department of Physics, University of Nevada, Reno, NV 8955\%, USA
}

(Dated: November 1, 2018)

\begin{abstract}
Ponderomotive energy shifts experienced by Rydberg atoms in optical fields are known to be well approximated by the classical quiver energy of a free electron. We examine such energy shifts quantum mechanically and elucidate how they relate to the ponderomotive shift of a free electron in off-resonant fields. We derive and evaluate corrections to the ponderomotive free electron polarizability in the length and velocity (transverse or Coulomb) gauges, which agree exactly as mandated by the gauge invariance. We also show how the free electron value emerges from the Dirac equation through summation over the Dirac sea states. We find that the free-electron AC Stark shift comes as an expectation value of a term proportional to the square of the vector potential in the velocity gauge. On the other hand, the same dominant contribution can be obtained to first order via a series expansion of the exact energy shift from the second order perturbation theory in the length gauge. Finally, we numerically examine the validity of the free-electron approximation. The correction to the free-electron value becomes smaller with increasing principal quantum number, and it is well below a per cent for 60 s states of $\mathrm{Rb}$ and $\mathrm{Sr}$ away from the resonances.
\end{abstract}

PACS numbers: 37.10.Jk, 32.10.Dk, 32.80.Qk, 32.80.Ee

Many of the prominent schemes for realizing quantum logic gates for quantum computing [1] 3 ], and studying few- and many-body physics via quantum simulation experiments using cold Rydberg atoms rely on optically trapped Rydberg atoms [4, 5]. Characterizing optical potentials experienced by Rydberg atoms plays an essential role in experimental realization of these schemes [6] .

The trapping AC Stark shift seen by the Rydberg atom is proportional to its dynamic polarizability, which is essentially that of a free electron quivering in the laser field. The free electron polarizability $\alpha_{\mathrm{e}}(\omega)=-e^{2} /\left(m_{e} \omega^{2}\right)$, where $\omega$ is the field frequency, can be derived from classical arguments by time averaging the electron kinetic energy in the oscillating electric field [7]. It can be also obtained quantum mechanically through the first order perturbation theory, with the perturbation being proportional to the intensity [8].

In this paper, we examine the applicability of the free electron approximation for Rydberg atoms in length and velocity gauges to gain insight as to how the exact $\mathrm{AC}$ Stark shift relates to the free electron value in both gauges. We show that $\alpha_{\mathrm{e}}(\omega)$ emerges from a term in the Hamiltonian proportional to the square of the vector potential $\left(A_{\mathrm{VG}}\right)^{2}$ in the velocity gauge, whereas it can be recovered to the leading order from a series expansion of the exact energy shift from the second order perturbation theory in the length gauge. We compute the corrections to the free electron polarizability and conclude that the correction to $\alpha_{\mathrm{e}}(\omega)$ in Rydberg states is relatively small away from resonances. Our discussion elucidates that rather than performing an exact sum over states calculation to evaluate the Rydberg state polarizabilities in the length gauge, it is simpler to calculate it as an expectation value of $\left(A_{\mathrm{VG}}\right)^{2}$. Throughout this paper, we assume plane wave electromagnetic fields rather than standing wave optical traps. Notice that otherwise the optical trap intensity variation can substantially modulate the Rydberg electron polarizability [9].

The paper is organized as follows: In the next section, we start by reviewing scalar and vector potentials of traveling waves in the length and velocity gauges. In section II, we derive AC Stark shifts and polarizabilities in both gauges within the non-relativistic formalism, and examine how they reduce to the free electron value. We then derive the free electron polarizability from the fullyrelativistic Dirac Hamiltonian in section III, and find that it comes from summation over the negative energy states in second order perturbation theory. We conclude in section IV by presenting numerical results illustrating the errors made by approximating the Rydberg state polarizabilities by the free electron values in the case of $\mathrm{Rb}$ and $\mathrm{Sr}$ atoms. We use atomic units throughout this report unless specifically stated otherwise.

\section{SCALAR AND VECTOR POTENTIALS}

Electromagnetic fields can be expressed in terms of the scalar and the vector potentials $\phi$ and $\mathbf{A}$. While the physical fields are unique, there is a certain degree of freedom in the choice of the potentials. Specifically, electric and magnetic fields in Gaussian units are $\mathbf{F}=-\nabla \phi-(\partial \mathbf{A} / \partial t) / c$ and $\mathbf{B}=\nabla \times \mathbf{A}$. A gauge transformation

$$
\begin{aligned}
\phi & \rightarrow \phi-\frac{1}{c} \frac{\partial \chi}{\partial t} \\
\mathbf{A} & \rightarrow \mathbf{A}+\nabla \chi
\end{aligned}
$$

leaves the physical quantities $\mathbf{F}$ and $\mathbf{B}$ unchanged, if $\chi$ satisfy the Lorentz condition [10]. The gauging function $\chi$ allows us to transform between different representa- 
tions of $\mathbf{A}$ and $\phi$ in different gauges, although the physical quantities, e.g. observables, are gauge invariant.

The Hamiltonian for an optical electron in external electromagnetic field may be written as

$$
H=\frac{1}{2}(\mathbf{p}-\mathbf{A} / c)^{2}+V_{C}+\phi
$$

where $V_{C}$ is the core potential seen by the electron.

In our current discussion, we treat the electromagnetic field as a plane wave propagating along the $z$-direction, and describe the electric field as $\mathbf{F}=F_{0} \hat{\epsilon} e^{\mathrm{i}(k z-\omega t)}+$ c.c.. In the velocity gauge (also known as the transverse or Coulomb gauge),

$$
\begin{aligned}
\mathbf{A}_{\mathrm{VG}} & =-\mathrm{i} \frac{F_{0} c}{\omega} \hat{\epsilon} e^{\mathrm{i}(k z-\omega t)}+\text { c.c. } \\
\phi_{\mathrm{LG}} & =0 .
\end{aligned}
$$

The length and the velocity gauges are related through the transformation function $\chi=-\mathbf{r} \cdot \mathbf{A}_{\mathrm{VG}}$, leading to potentials in the length gauge [10]:

$$
\begin{aligned}
\mathbf{A}_{\mathrm{LG}} & =-F_{0} \hat{\mathbf{k}}(\hat{\epsilon} \cdot \mathbf{r}) e^{\mathrm{i}(k z-\omega t)}+\text { c.c. }, \\
\phi_{\mathrm{LG}} & =-F_{0}(\hat{\epsilon} \cdot \mathbf{r}) e^{\mathrm{i}(k z-\omega t)}+\text { c.c.. }
\end{aligned}
$$

\section{STARK SHIFTS AND POLARIZABILITIES IN THE NON-RELATIVISTIC APPROXIMATION}

\section{A. Velocity Gauge}

In the velocity gauge (4), the Hamiltonian becomes,

$$
H=\frac{p^{2}}{2}+V_{C}-\frac{\mathbf{A}_{\mathrm{VG}} \cdot \mathbf{p}}{c}+\frac{\left(A_{\mathrm{VG}}\right)^{2}}{2 c^{2}} .
$$

We calculate the energy shifts within the Floquet formalism of quasi-energy states [11] for the third term, and use the first order perturbation theory for the fourth term since it is already second order in the field strength. The energy shift for a Rydberg state $|r\rangle$ therefore becomes

$$
\begin{gathered}
\delta E_{\mathrm{VG}}(\omega)=\frac{1}{4 c^{2}} \sum_{j} \frac{2 \Delta E_{j}}{\Delta E_{j}^{2}-\omega^{2}}\left|\left\langle r\left|\mathbf{A}_{\mathrm{VG}} \cdot \mathbf{p}\right| j\right\rangle\right|^{2} \\
+\left\langle r\left|\left(A_{\mathrm{VG}}\right)^{2}\right| r\right\rangle /\left(2 c^{2}\right) .
\end{gathered}
$$

The first term can be decomposed into the scalar, vector and tensor contributions. We focus on the dominant scalar contribution to the Stark shift and arrive at

$$
\delta E_{\mathrm{VG}}(\omega)=\frac{F_{0}^{2}}{4 \omega^{2}}+\frac{F_{0}^{2}}{4 \omega^{2}} \sum_{j} \frac{2 \Delta E_{j}^{3}}{\Delta E_{j}^{2}-\omega^{2}} \frac{1}{3}|\langle r|\mathbf{D}| j\rangle|^{2},
$$

where $E_{r}-E_{j}=\Delta E_{j}$, and we used

$$
\langle r|\hat{\epsilon} \cdot \mathbf{p}| j\rangle=-\mathrm{i} \Delta E_{j} \hat{\epsilon}\langle r|\mathbf{D}| j\rangle .
$$

Here $\mathbf{D}$ is the usual dipole operator. The summation over the magnetic quantum number can be evaluated explicitly using the Wigner-Eckart theorem:

$$
\sum_{\lambda, M_{j}}(-1)^{\lambda}\left\langle r\left|\mathbf{D}_{\lambda}\right| j\right\rangle\left\langle j\left|\mathbf{D}_{-\lambda}\right| r\right\rangle=\frac{1}{2 l_{r}+1} \sum_{n_{j}, l_{j}}|\langle r\|D\| j\rangle|^{2}
$$

where $\langle r\|D\| j\rangle$ are the reduced dipole matrix elements.

The first term in Eq. (7) can be immediately identified as the ponderomotive energy shift for a free electron quivering in electromagnetic field. Notice that it came directly from the $A_{\mathrm{VG}}^{2}$ term in the Hamiltonian (6), and it is the dominant term for a Rydberg state. By contrast, the second term in (7) is the correction to the free electron ponderomotive shift. All of the resonance structure is included in the second correction term, whereas the free electron shift only provides a smooth background on which the resonance structure sits.

We can express $\delta E_{\mathrm{VG}}$ in terms of the conventional $\mathrm{AC}$ polarizability through its definition for the plane waves, $\delta E(\omega)=-\alpha(\omega) F_{0}^{2} / 4$. Then the AC polarizability in the velocity gauge becomes

$$
\alpha_{\mathrm{VG}}(\omega)=\alpha_{\mathrm{e}}(\omega)-\frac{2}{3 \omega^{2}} \sum_{j} \frac{\Delta E_{j}^{3}}{\Delta E_{j}^{2}-\omega^{2}}|\langle r|\mathbf{D}| j\rangle|^{2} .
$$

Here $\alpha_{\mathrm{e}}(\omega)=-1 / \omega^{2}$ is the free electron polarizability.

\section{B. Length Gauge}

We now derive Eq. (9) in the length gauge, and thereby establish the gauge invariance. Using the length gauge vector and scalar potentials (5), the Hamiltonian can be expressed as

$$
H=\frac{p^{2}}{2}+V_{C}-\frac{\mathbf{A}_{\mathrm{LG}} \cdot \mathbf{p}}{c}+\frac{\left(A_{\mathrm{LG}}\right)^{2}}{2 c^{2}}+\phi_{\mathrm{LG}} .
$$

To calculate the energy shifts resulting from the last three terms in the Hamiltonian, we again use the quasi-energy formalism [11] for the third and the fifth terms, and the first order perturbation theory for the fourth term:

$$
\begin{aligned}
\delta E_{\mathrm{LG}}(\omega)=\frac{F_{0}^{2}}{4} & \sum_{j} \frac{2 \Delta E_{j} / 3}{\Delta E_{j}^{2}-\omega^{2}}\left|\left\langle r\left|\mathbf{A}_{\mathrm{LG}} \cdot \mathbf{p} / c+\phi_{\mathrm{LG}}\right| j\right\rangle\right|^{2} \\
& +\frac{F_{0}^{2}}{2 c^{2}}\left\langle r\left|\left(A_{\mathrm{LG}}\right)^{2}\right| r\right\rangle
\end{aligned}
$$

Upon expanding the square, we encounter three contributions to the first term. Out of the three, the dominant one is the $\left|\left\langle r\left|\phi_{\mathrm{LG}}\right| j\right\rangle\right|^{2}$ term. The term involving $\left|\left\langle r\left|\mathbf{A}_{\mathrm{LG}} \cdot \mathbf{p}\right| j\right\rangle\right|^{2}$, and the last term involving $\left(A_{\mathrm{LG}}\right)^{2}$ are suppressed by a factor of $1 / c^{2} \sim 10^{-4}$ and can be safely ignored in the non-relativistic approximation. The cross term between the $\mathbf{A}_{\mathrm{LG}} \cdot \mathbf{p}$ and $\phi_{\mathrm{LG}}$ drops out because it is proportional to $\operatorname{Re}\left[\left\langle r\left|\mathbf{A}_{\mathrm{LG}} \cdot \mathbf{p}\right| j\right\rangle\left\langle j\left|\left(\phi_{\mathrm{LG}}\right)^{\dagger}\right| r\right\rangle\right]^{2}$ which vanishes on the account of $\left\langle r\left|\mathbf{A}_{\mathrm{LG}} \cdot \mathbf{p}\right| j\right\rangle$ being purely imaginary (due to $\langle r|\hat{\epsilon} \cdot \mathbf{p}| j\rangle=-\mathrm{i} \Delta E_{j} \hat{\epsilon}\langle r|\mathbf{D}| j\rangle$ ). We again 
focus on the dominant scalar contribution to the Stark shift, which leaves us with

$$
\delta E_{\mathrm{LG}}(\omega)=\frac{F_{0}^{2}}{2} \frac{1}{3} \sum_{j} \frac{\Delta E_{j}}{\Delta E_{j}^{2}-\omega^{2}}|\langle r|\mathbf{D}| j\rangle|^{2} .
$$

Through the definition $\delta E(\omega)=-\alpha(\omega) F_{0}^{2} / 4$, the polarizability in the length gauge can be written as:

$$
\alpha_{\mathrm{LG}}(\omega)=-\frac{2}{3} \sum_{j} \frac{\Delta E_{j}}{\Delta E_{j}^{2}-\omega^{2}}|\langle r|\mathbf{D}| j\rangle|^{2} .
$$

To reveal how this expression relates to $\alpha_{\mathrm{e}}(\omega)$ and the polarizability in the velocity gauge, we expand the resolvent operator in Eq. (13) in powers of $\Delta E_{j} / \omega$ :

$$
\frac{\Delta E_{j}}{\Delta E_{j}^{2}-\omega^{2}}=\frac{\Delta E_{j}}{\omega^{2}}\left(-1-\frac{\Delta E_{j}^{2}}{\omega^{2}}-\frac{\Delta E_{j}^{4}}{\omega^{4}}-\cdots\right) .
$$

This results in a series expansion of the polarizability in the length gauge:

$$
\begin{aligned}
\alpha_{\mathrm{LG}}(\omega)= & \frac{2}{3 \omega^{2}} \sum_{j} \Delta E_{j}|\langle r|\mathbf{D}| j\rangle|^{2} \\
& +\frac{2}{3 \omega^{4}} \sum_{j} \Delta E_{j}^{3}|\langle r|\mathbf{D}| j\rangle|^{2} \\
& +\frac{2}{3 \omega^{6}} \sum_{j} \Delta E_{j}^{5}|\langle r|\mathbf{D}| j\rangle|^{2}+\cdots .
\end{aligned}
$$

This series can be resummed such that the first term is separated out,

$$
\begin{aligned}
\alpha_{\mathrm{LG}}(\omega)= & \frac{2}{3 \omega^{2}} \sum_{j} \Delta E_{j}|\langle r|\mathbf{D}| j\rangle|^{2} \\
& +\frac{2}{3 \omega^{2}} \sum_{k}\left(\sum_{j} \frac{\Delta E_{j}^{3+2 k}}{\omega^{2+2 k}}|\langle r|\mathbf{D}| j\rangle|^{2}\right) .
\end{aligned}
$$

With the help of the oscillator sum rule,

$$
-\frac{2}{3} \sum_{j} \Delta E_{j}|\langle r|\mathbf{D}| j\rangle|^{2}=1
$$

we recover the free electron polarizability from the first term in (15) when summed over a complete set of states (note that $\Delta E_{j}=-\left(E_{j}-E_{r}\right)$ ). On the other hand, with the aid of the expansion (14), the outer sum in the second term can be collapsed back into the same correction term to the free electron polarizability in the velocity gauge (9). Thus

$$
\alpha_{\mathrm{LG}}(\omega)=\alpha_{\mathrm{e}}(\omega)+\frac{2}{3 \omega^{4}} \sum_{j} \frac{\Delta E_{j}^{3}}{\Delta E_{j}^{2}-\omega^{2}}|\langle r|\mathbf{D}| j\rangle|^{2},
$$

and $\alpha_{\mathrm{LG}}(\omega) \equiv \alpha_{\mathrm{VG}}(\omega)$. This expression for the length gauge polarizability is identical to the one in Eq. (9) in the velocity gauge, confirming the equivalence of the $\mathrm{AC}$ Stark shifts in both gauges, i.e. the gauge invariance. These expressions also show how the free electron term originates, and we will demonstrate below that the correction to $\alpha_{e}(\omega)$ is indeed very small for Rydberg states.

In Ref. [12], the emergence of the free electron polarizability in the length gauge has been shown numerically for Rydberg states of Rb atom. Ref. [13] has provided an alternative analytical expression using higher rank oscillator sum rules. However, we find that the formula of Ref. 13] has poor convergence properties when evaluated numerically.

\section{DIRAC SEA}

So far we examined the AC Stark effect in the nonrelativistic formalism. In the velocity gauge the dominant contribution came from the expectation value of the vector-potential-squared term $A_{\mathrm{VG}}^{2}$. Curiously, the fully-relativistic Dirac Hamiltonian involves only linear couplings to electromagnetic fields, thereby such term is missing in the lowest-order of perturbation theory. This naturally raises a question of just how the free-electron polarizability emerges from the relativistic equations. As we show in this Section, it comes from summations over the negative energy (positron, $E_{n}<-m_{e} c^{2}$ ) states, i.e. from the Dirac sea. Similarly pronounced effects of Dirac sea on weak atomic transition amplitudes were found earlier [14, 15] in relativistic many-body calculations.

We start with the coupling to electromagnetic fields

$$
V=\alpha \cdot \mathbf{A}(\mathbf{r}, t)-\phi(\mathbf{r}, t),
$$

where $\alpha$ are the conventional Dirac matrices. In the velocity gauge $\phi_{\mathrm{VG}}(\mathbf{r}, t)=0$, so that

$$
V=\alpha \cdot \mathbf{A}_{\mathrm{VG}}(\mathbf{r}, t) .
$$

Applying the second-order Floquet formalism, we arrive at the fully-relativistic AC polarizability

$$
\begin{aligned}
\alpha_{\mathrm{VG}, \operatorname{Dirac}}(\omega)=-\frac{2 c^{2}}{\omega^{2}} \sum_{j} & \frac{\left(E_{r}-E_{j}\right)}{\left(E_{r}-E_{j}\right)^{2}-\omega^{2}} \\
& \times\left|\left\langle\psi_{r}|\alpha \cdot \hat{\epsilon}| \psi_{j}\right\rangle\right|^{2} .
\end{aligned}
$$

The summation over intermediate states in the above equation spans the complete spectrum of the Dirac equation, i.e., both positive and negative energy states. It can be shown using the Pauli approximation that the sum over the conventional positive energy states recovers the second term (second-order sum) in the nonrelativistic expression for the AC polarizability. Now we demonstrate that the dominant, free-electron polarizability term, emerges from summing over the negative energy states. In this case, the Rydberg electron energy $E_{r}$ is above the Dirac sea level and $\left(E_{r}-E_{j}\right) \approx 2 m_{e} c^{2}$ and $\left(E_{r}-E_{j}\right) \gg \omega$ (we naturally assume that the photon 
energy is well below $\left.2 m_{e} c^{2}\right)$. Within this approximation, the dynamic AC polarizability becomes

$$
\begin{aligned}
\alpha_{\mathrm{VG}, \operatorname{Dirac}}^{(-)}(\omega)=- & \frac{2 c^{2}}{\omega^{2}\left(2 m_{e} c^{2}\right)} \\
& \times \sum_{E_{j}<-m_{e} c^{2}}\left\langle\psi_{r}|\alpha \cdot \hat{\epsilon}| \psi_{j}\right\rangle\left\langle\psi_{j}|\alpha \cdot \hat{\epsilon}| \psi_{r}\right\rangle .
\end{aligned}
$$

The Dirac matrices $\alpha$ mix the large and the small components of the Dirac bi-spinor, $\psi=\left(\begin{array}{c}\psi^{l} \\ \psi^{s}\end{array}\right)$. Part of the summation on the right hand side of (21) can therefore be written as

$$
\sum_{E_{j}<-m_{e} c^{2}}\left\langle\psi_{r}^{l}|\sigma \cdot \hat{\epsilon}| \psi_{j}^{s}\right\rangle\left\langle\psi_{j}^{s}|\sigma \cdot \hat{\epsilon}| \psi_{r}^{l}\right\rangle .
$$

In the non-relativistic approximation, the negative energy states are complete amongst themselves, i.e. $\sum_{b}\left|\psi_{b}^{s}\right\rangle\left\langle\psi_{b}^{s}\right| \approx 1$, leading to

$$
\alpha_{\mathrm{VG}, \operatorname{Dirac}}^{(-)}(\omega)=-\frac{1}{\omega^{2}}\left\langle\psi_{a}^{l}|\sigma \cdot \hat{\epsilon}| \psi_{a}^{l}\right\rangle=-\frac{1}{\omega^{2}}
$$

where we used $(\sigma \cdot \hat{\epsilon})^{2}=1$ and $m_{e}=1$ a.u.. Therefore we recover the free electron polarizability in atomic units. Is not interesting that describing purely classical effect of electron quiver motion requires the notion of anti-mater?

\section{CORRECTION TO THE FREE ELECTRON POLARIZABILITY}

In all our calculations below, we use the single active electron approximation, and represent the atomic core with the model potential using the correct quantum defects for Rb and $\mathrm{Sr}$ atoms from [16]:

$$
V_{C}=-\frac{1+2 e^{-\alpha_{1} r}+r \alpha_{2} e^{-\alpha_{3} r}}{r}-\frac{\alpha_{s}}{2 r^{4}}\left[1-\exp \left(-r^{3}\right)\right]^{2},
$$

where $\alpha_{1}, \alpha_{2}$ and $\alpha_{3}$ are $l$-dependent screening parameters, and $\alpha_{s}$ is the static polarizability of the core [17]. For illustrative purposes, we pick $\mathrm{Rb}$ and $\mathrm{Sr}$ atoms because they have very different core polarizabilities. For $\mathrm{Rb}$, the static core polarizability is $\sim 9$ a.u., whereas the polarizability for the $\mathrm{Sr}^{+}$ion with a 5 s valence electron serving as spectator is $\sim 91$ a.u., an order of magnitude larger. This results in large differences in quantum defects $\delta_{l}$ for the $s$ Rydberg states: for example in the $100 \mathrm{~s}$ state of $\mathrm{Rb} \delta_{0} \simeq 3.28$ and for $\mathrm{Sr} \delta_{0} \simeq 5.02$. In our calculations, we assume that the $\mathrm{Sr}$ atom is in a $J=0$ state, and we ignore contributions from the resonant structure from the $5 \mathrm{~s}$ valence electron.

In order to evaluate polarizabilities in both the length and the velocity gauges, we have to evaluate sums over a complete set of states in (9) and (13). We check the completeness of our basis using the well known oscillator sum rule. We find,

$$
\begin{aligned}
& -\frac{2}{3} \sum_{j} \Delta E_{j}|\langle r|\mathbf{D}| j\rangle|^{2}=0.967 \quad(\mathrm{Rb}), \\
& -\frac{2}{3} \sum_{j} \Delta E_{j}|\langle r|\mathbf{D}| j\rangle|^{2}=0.989 \quad(\mathrm{Sr}),
\end{aligned}
$$

while summing over $p$ states with $n=2$ through 200 for the 100s states of the $\mathrm{Rb}$ and $\mathrm{Sr}$ atoms.
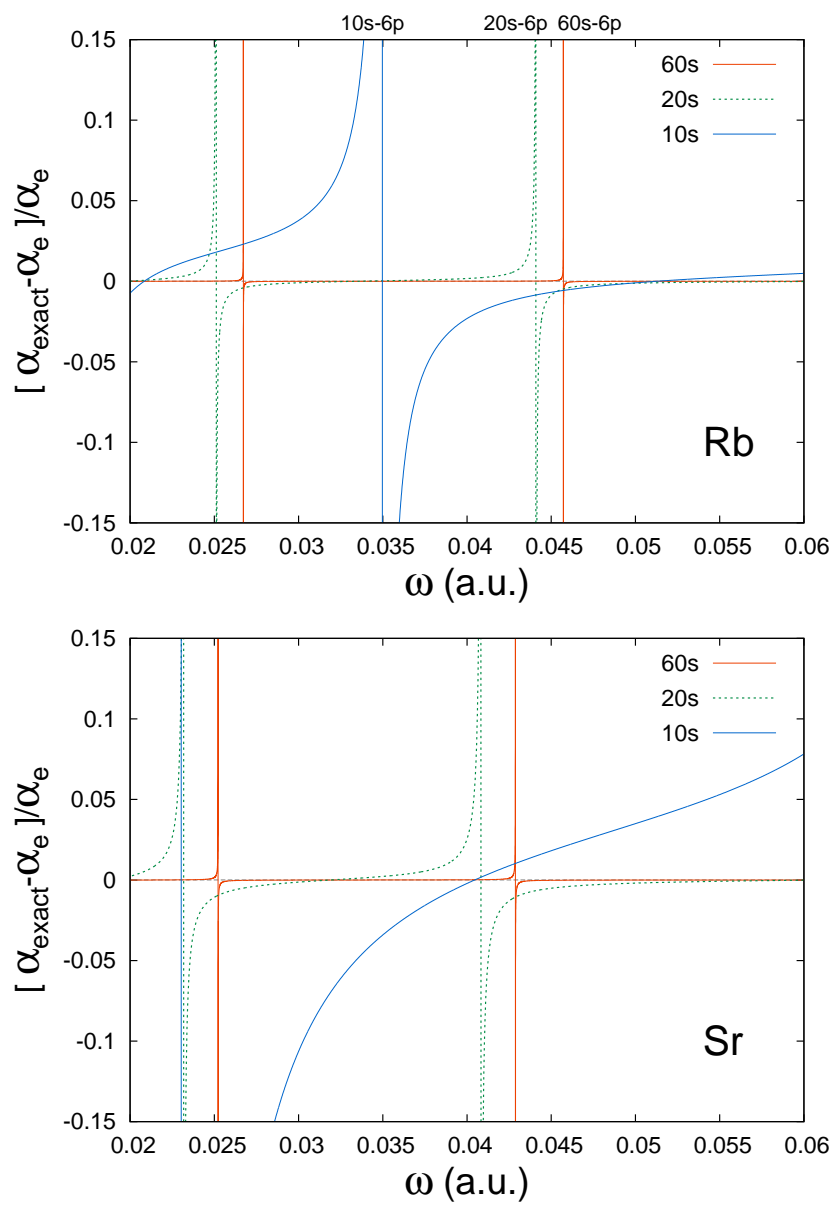

FIG. 1: (Color Online) The fractional corrections to the free electron polarizability for the $60 \mathrm{~s}, 20 \mathrm{~s}$ and $10 \mathrm{~s}$ states of $\mathrm{Rb}$ and $\mathrm{Sr}$ atoms in the IR region of the spectrum. The resonance structure is entirely contained within these corrections, and the resonances become wider for higher $n$ s states. The $\omega$ axis spans wavelengths between $\sim 2300 \mathrm{~nm}$ and $\sim 760 \mathrm{~nm}$.

We have shown that the Rydberg state polarizability in the length (17) and velocity gauges (9) are identical as a result of the gauge invariance, i.e. $\alpha_{\mathrm{LG}}(\omega) \equiv \alpha_{\mathrm{VG}}(\omega)$. Furthermore, we have expressed these exact polarizabilities as a sum of the free electron polarizability $\alpha_{\mathrm{e}}(\omega)$ and an exact correction. The resonance structure of the polarizabilities is entirely captured by this correction term, whereas $\alpha_{\mathrm{e}}(\omega)$ only provides a smooth background. The 
fractional corrections for various $n$ s states of $\mathrm{Rb}$ and $\mathrm{Sr}$ are shown in Fig. 1 in the IR region of the spectrum. The resonant structure is evident in the plots, and the widths of the resonances increase with increasing principal quantum number. Also, the sizes of the corrections grow smaller as $n$ is increased meaning that the near-free electron approximation to the Rydberg electron polarizability gradually becomes more accurate away from the resonances. With the exception of the 10s state, the corrections are well below a per cent for both $\mathrm{Rb}$ and $\mathrm{Sr}$, except at the resonances.

Comparing the upper and lower panels of Fig. 1, we observe that the errors made by approximating $\alpha_{\mathrm{LG}}(\omega)$ and $\alpha_{\mathrm{VG}}(\omega)$ by $\alpha_{\mathrm{e}}(\omega)$ are larger for Sr than for Rb. This stems from the larger static polarizability of the $\mathrm{Sr}^{+}$ion with a $5 \mathrm{~s}$ valence electron compared to the $\mathrm{Rb}^{+}$ionic core, a property which is contained in the model potential (24). Although the static core polarizabilities for these atoms differ by an order of magnitude, the corrections to $\alpha_{\mathrm{e}}(\omega)$ are still much less than a percent in both cases, except in the immediate vicinity of resonances.

Another feature seen in Fig. 1 is that the widths of the resonances decrease with increasing principal quantum number. For example, for $\mathrm{Rb}$ the width of the $20 \mathrm{~s}-6 \mathrm{p}$ resonance is significantly larger than that of the $60 \mathrm{~s}-6 \mathrm{p}$ resonance. On the other hand, the $10 \mathrm{~s}-6 \mathrm{p}$ resonance is so large that the correction to $\alpha_{\mathrm{e}}(\omega)$ for the 10s state of $\mathrm{Rb}$ is at least a couple of per cent nearly for all frequencies spanned in Fig. 1. Similar observations can also be made for Sr. The widening of these resonances with increasing $n$ can be qualitatively understood if one realizes that the widths of the resonances in Eqs. (9) and (17) are controlled by the square of the dipole matrix elements, which scales as $|\langle r|D| j\rangle|^{2} \sim 1 / n^{3}$.

Ref. [9] found strong intensity landscape modulations of the effective polarizability for Rydberg atoms trapped in IR lattices. Because of the low frequency of the trapping field, the Rydberg electron polarizability can potentially deviate quite substantially from the free electron value. This is because at sufficiently low frequencies the polarizability must approach its static limit, whereas the free electron value diverges. In this paper, we have shown that the free electron approximation holds, even at IR wavelengths of thousands of $\mathrm{nm}$.

This work was supported by the NSF Grant No. PHY1212482.
[1] D. Jaksch, J. I. Cirac, P. Zoller, S. L. Rolston, R. Côtè, and M. D. Lukin, Phys. Rev. Lett. 85, 2208 (2000)

[2] E. Urban, T. A. Johnson, T. Henage, L. Isenhower, D. D. Yavuz, T. G. Walker, and M. Saffman, Nature Physics 5, $110(2009)$

[3] A. Gaetan, Y. Miroshnychenko, T. Wilk, A. Chotia, M. Viteau, D. Comparat, P. Pillet, A. Browaeys, and P. Grangier, Nature Physics 5, 115 (2009)

[4] Hendrik Weimer, Markus Müller, Igor Lesanovsky, Peter Zoller and Hans Peter Büchler, Nature Physics 6, 382 (2010)

[5] I. Bloch, J. Dalibard, and S. Nascimbène, Nature Physics 8, 267 (2012)

[6] M. Saffman, T. Walker, and K. Molmer., Rev. Mod. Phys. 82, 2313 (2010)

[7] K. C. Younge, S. E. Anderson, and G. Raithel, New J. Phys. 12, 023031 (2010)

[8] S. Zhang, F. Robicheaux, and M. Saffman, Phys. Rev. A 84, 043408 (2011)
[9] T. Topcu and A. Derevianko, arXiv:1305.6570 [physics.atom-ph] (2013)

[10] J. D. Jackson, Classical Electrodynamics, John Wiles \& Sons, Inc. Third Edition (1998)

[11] N. L. Manakov, V. D. Ovsiannikov, nd L. P. Rapoport, Phys. Rep. 141, 319 (1986)

[12] M. S. Safronova, C. J. Williams, and C. W. Clark, Phys. Rev. A 67, 040303(R) (2003)

[13] V. D. Ovsiannikov, Opt. Spectrosc. (USSR) 49, 1 (1980)

[14] A. Derevianko, I. M. Savukov, W. R. Johnson, and D. R. Plante, Phys. Rev. A 58, 4453 (1998)

[15] I. M. Savukov, A. Derevianko, H. G. Berry, W. R. Johnson, Phys. Rev. Lett. 83, 2914 (1999)

[16] M. Aymar, C. H. Greene, and E. Luc-Koenig, Rev. Mod. Phys. 68, 1015 (1996)

[17] B. K. Sahoo, R. G. E. Timmermans, B. P. Das, and D. Mukherjee, Phys. Rev. A 80, 062506 (2009) 